ARTÍ́CULO ORIGINAL

\title{
Calidad de vida y disfunción eréctil en pacientes con hiperplasia prostática benigna
}

\author{
Quality of life and erectile dysfunction in patients with benign prostatic hyperplasia
}

Álvaro J. Montiel-Jarquín ${ }^{*}$, Claudia T. Gutiérrez-Quiroz, Ana L. Pérez-Vázquez¹, Johny J. Ortiz-Agustín', Arturo García-Galicia ${ }^{1}$ y Jorge Loría-Castellanos ${ }^{2}$

'Unidad Médica de Alta Especialidad Hospital de Especialidades de Puebla, Instituto Mexicano del Seguro Social (IMSS); ${ }^{2}$ División de Proyectos Especiales en Salud, IMSS. Puebla, Puebla, México

\section{Resumen}

Antecedentes: La patología de la próstata más frecuente es la hiperplasia prostática benigna. A los 50 años, el 50\% de los hombres son diagnosticados y a los 80 años aumenta hasta al 90\%. La prevalencia de disfunción eréctil en conjunto con hiperplasia prostática benigna es del 5.2-40\%, y los pacientes con hiperplasia prostática benigna es 1.33-6.24 veces más frecuente que tengan disfunción eréctil que aquellos sin hiperplasia prostática benigna. Ambas afecciones repercuten en la calidad de vida. Objetivo: Identificar la calidad de vida y el grado de disfunción eréctil en pacientes con hiperplasia prostática benigna. Método: Estudio transversal, descriptivo, en pacientes con crecimiento prostático benigno a los cuales se les aplicaron dos cuestionarios: SF-12 para calidad de vida e Índice Internacional de Función Eréctil versión 5 para disfunción eréctil. Resultados: Fueron 101 pacientes, edad media de $66.5 \pm 8.5$ años, promedio físico de 38.68, promedio mental de 43.35, 14 de ellos sin disfunción eréctil y 38 con disfunción eréctil leve, 33 leve a moderada, 15 moderada y 1 grave. Conclusiones: Los pacientes con hiperplasia prostática benigna tienen una salud física mala y una salud mental buena. El $70.3 \%$ de los pacientes muestran grado leve o leve-moderado de disfunción eréctil.

Palabras clave: Hiperplasia prostática benigna. Disfunción eréctil. Calidad de vida.

\begin{abstract}
Background: The most frequent prostatic pathology is benign prostatic hyperplasia. By the age of $50,50 \%$ are diagnosed with it and by the age of 80 , it increases to $90 \%$. The erectile dysfunction prevalence along benign prostatic hyperplasia is 5.2\%-40\%. Patients with benign prostatic hyperplasia are 1.33-6.24 times more likely to have erectile dysfunction tan without benign prostatic hyperplasia. Objective: To identify quality of life and to grade erectile dysfunction in patients with benign prostatic hyperplasia. Method: A transversal, descriptive study was conducted in patients with benign prostatic hyperplasia. Two questionnaires were applied: SF-12 for quality of life and IIEF-5 for erectile dysfunction. Results: 101 patients, mean age $66.5 \pm 8.5$ years, mean physical aspect 38.68, mean mental aspect 43.35, 14 without erectile dysfunction, 38 mild grade, 33 mild to moderate, 15 moderate and 1 severe. Conclusions: Benign prostatic hyperplasia patients have poor physical health and good mental health. $70.3 \%$ of patients have a grade of erectile dysfunction between mild and mild-moderate.
\end{abstract}

Key words: Life quality. Erectile disfunction. Benign prostatic hyperplasia.

Correspondencia:

*Arturo García-Galicia

2 Norte 2004
Centro

C.P. 72000 , Puebla, Pue., México

E-mail: arturo.garciaga@imss.gob.mx

0009-7411/@ 2020 Academia Mexicana de Cirugía. Publicado por Permanyer. Este es un artículo open access bajo la licencia CC BY-NC-ND (http://creativecommons.org/licenses/by-nc-nd/4.0/).

Cir Cir. 2021;89(2):218-222

Contents available at PubMed www.cirugiaycirujanos.com
la licencia CC BY-NC-ND 


\section{Introducción}

La disfunción eréctil (DE) es la imposibilidad de conseguir o mantener una erección suficiente para una actividad sexual satisfactoria. La prevalencia de hiperplasia prostática benigna (HPB) con DE es del 5.2 al $40 \%$, y los pacientes con HPB reportan 1.33 a 6.24 veces más DE que aquellos sin HPB. Asimismo, los pacientes que muestran síntomas urinarios bajos (SUB) tienen una disminución en la frecuencia y en la satisfacción de la actividad sexual'.

Algunos estudios han demostrado que los pacientes con HPB y DE muestran dificultades sociales y emocionales, como ansiedad, depresión, aislamiento social y dolor que puede resultar en fatiga, disminución de la vitalidad e insomnio, con disminución de la calidad de vida ${ }^{2,3}$. Por otra parte, la prevalencia de DE es dependiente de la edad y se incrementa del $5 \%$ en los pacientes de 40 años al $15 \%$ en los mayores de 70 años. Para la edad de 50 años, el $50 \%$ son diagnosticados, y hacia los 80 años aumentan hasta el $90 \%$. La escala IIEF-5 (Índice Internacional de Función Eréctil versión 5) demuestra una relación fuerte entre los SUB y la DE; en los pacientes de 50-59 años, la IIEF-5 fue de 22.3 sin SUB y de 14.9 con SUB ${ }^{4,5}$.

El crecimiento glandular de la HPB crea una compresión sobre la uretra, resultando en SUB como nicturia, urgencia miccional, poliuria, sensación de vaciado incompleto, tenesmo vesical y disminución del calibre y de la fuerza del chorro urinario ${ }^{6}$. Los mecanismos fisiopatológicos por los que la HPB causa SUB son: reducción de óxido nítrico, señalización de monofosfato cíclico de guanosina, inflamación crónica/desbalance de hormonas esteroideas e incremento de la actividad RhoA-Rho-Kinasa, hiperactividad autonómica y ateroesclerosis pélvica. Estos factores disminuyen la función de los nervios y del endotelio, ocasionan insuficiencia arterial, reducen el flujo sanguíneo, producen lesión tisular por hipoxia, aumentan la proliferación de músculo liso en la próstata y causan hipertrofia vesical. Todos estos resultados afectan la función eréctil por reducción en la inervación y disminución de la relajación del músculo liso en los cuerpos cavernosos ${ }^{7-9}$.

El tratamiento médico integral se basa en:

- Inhibidores de la $5 \alpha$ reductasa: inhiben esta enzima implicada en la transformación de testosterona a dihidrotestosterona. La finasterida es un inhibidor selectivo de tipo $25 \alpha$ reductasa y disminuye las concentraciones de dihidrotestosterona aproximadamente un $60-70 \%$. Los efectos adversos en la salud sexual son DE persistente, pérdida de libido, disminución del volumen de eyaculación y depresión?.

- Bloqueadores alfa: son bloqueadores selectivos de los receptores $\alpha 1$ en la próstata y el cuello vesical, que producen una relajación del músculo liso y favorecen la micción. El fármaco más usado de esta familia es la tamsulosina. Los efectos adversos son eyaculación retrógrada, mareo, síncope e hipotensión ${ }^{10}$.

- Psicoterapia: se sugiere que todos los hombres bajo tratamiento reciban ayuda psicológica para reducir su ansiedad, mejorar su apego al tratamiento e incorporarse a su vida sexual. Además, se debe tener conocimiento de la posible comorbilidad que pudiera afectar de manera negativa la función eréctil, y hay que promover cambios en el estilo de vida y la dieta, e incrementar la actividad física para mejorar el estado de salud global y sexual ${ }^{4,11}$.

El IEEF-5 es un cuestionario validado ampliamente para población latinoamericana, que se utiliza para evaluar la función eréctil. Es breve, válido desde el punto de vista psicométrico y autoaplicable. En 15 reactivos evalúa la respuesta sexual humana (deseo, erección, eyaculación y orgasmo), la satisfacción en la relación sexual y la satisfacción global, con la puntuación siguiente: $22-25$ puntos, sin DE; 17-21 puntos, DE leve; 12-16 puntos, DE leve-moderada; 8-11 puntos, DE moderada; y 5-7 puntos, DE grave ${ }^{12}$.

La calidad de vida se considera un rubro fundamental en la atención de patologías crónicas diversas, como la HPB y la DE. El SF-12 proporciona un perfil del estado de salud en la población general a partir de 14 años de edad. Es un cuestionario autoadministrado, con respuesta en menos de 2 minutos. Consta de 12 ítems provenientes de las ocho dimensiones del SF-36: función física (dos ítems), función social (un ítem), rol físico (dos ítems), rol emocional (dos ítems), salud mental (dos ítems), vitalidad (un ítem), dolor corporal (un ítem) y salud general (un ítem) ${ }^{13}$.

El objetivo de este trabajo fue identificar la calidad de vida y el grado de DE en pacientes con HPB sin comorbilidad y que no se hubieran sometido a tratamiento quirúrgico, para enfatizar el efecto de la HPB en la DE, así como relacionarlo con la literatura mundial.

\section{Método}

Estudio transversal, descriptivo, realizado en pacientes con HPB que acudieron a la consulta externa 
de urología durante el periodo comprendido de julio a noviembre de 2019.

Se incluyeron pacientes mayores de 40 años con crecimiento prostático (definido como crecimiento de la próstata por examen digital o por ultrasonido en pacientes con "síntomas del tracto urinario inferior", de acuerdo con la Escala Internacional de Síntomas de la Próstata (I-PSS), quienes firmaron la carta de consentimiento informado. Se excluyeron pacientes con patología de origen neoplásico o infeccioso, comorbilidad que por sí misma afecte la calidad de vida o la DE (como diabetes mellitus, hipertensión arterial o dislipidemia) y pacientes sin pareja sexual, y se eliminaron los que no desearon participar en el estudio.

Para determinar la calidad de vida se aplicó el cuestionario SF-12 para analizar los ocho dominios mencionados, y para valorar el grado de disfunción eréctil se aplicó el cuestionario IIEF-5 para su evaluación global y las categorías ya descritas.

Se utilizó un muestreo consecutivo conveniente, con cálculo del tamaño de la muestra para poblaciones finitas, con un intervalo de confianza del $95 \%$ y un error tolerado del $5 \%$, arrojando una muestra de 89 pacientes.

Se utilizó estadística descriptiva y se midió la correlación entre la calidad de vida y la DE utilizando el coeficiente de Spearman. Se consideró estadísticamente significativo un valor de $p \leq 0.05$.

\section{Resultados}

De un total de 250 pacientes se excluyeron 149 por presentar alguna patología asociada que podía afectar la calidad de vida y la DE, por lo que quedaron 101 pacientes. La edad media fue de $66.5 \pm 8.5$ años (rango: 47-84).

Los puntajes medios obtenidos en el SF-12 para los dominios físico y mental fueron de 38.68 y 43.35 , respectivamente. Con respecto al grado de $D E, 38$ (37.6\%) pacientes se encontraron con DE leve; el resto de los detalles se muestra en la tabla 1.

En cuanto al tratamiento empleado en los pacientes, el $48.5 \%$ estaban en tratamiento con tamsulosina y el $12.9 \%$ no tenían tratamiento debido a que su diagnóstico era reciente. La frecuencia del resto de los tratamientos encontrados y los puntajes del IIEF-5 de acuerdo con el tratamiento empleado se muestran en la tabla 2.

La correlación entre el grado de DE y el aspecto mental de la calidad de vida no dio resultado
Tabla 1. Grado de disfunción eréctil

\begin{tabular}{lcc}
\hline & $\mathbf{n}$ & $\%$ \\
\hline $22-25$ = no hay DE & 14 & 13.9 \\
$17-21=$ DE leve & 38 & 37.6 \\
12-16 = DE leve-moderada & 33 & 32.7 \\
8-11 = DE moderada & 15 & 14.9 \\
5-7 = DE grave & 1 & 1.0 \\
Total & 101 & 100.0 \\
\hline DE. disfunción eréctil & &
\end{tabular}

DE: disfunción eréctil.

Tabla 2. Puntaje obtenido en el cuestionario IIEF-5 según el tratamiento para la hiperplasia prostática benigna establecido en los pacientes incluidos

\begin{tabular}{lccc}
\hline & \multicolumn{2}{c}{ Frecuencia } & Promedio IIEF-5 DE \\
\cline { 2 - 3 } & \multicolumn{2}{c}{ Pacientes } & $\%$ \\
\hline Tamsulosina & 49 & 48.5 & 16.48 \\
Finasterida & 2 & 2 & 18.5 \\
Tamsulosina + finasterida & 37 & 36.6 & 15.51 \\
Ninguno & 13 & 12.9 & 17.53 \\
Total & 101 & 100 & 16.30 \\
\hline
\end{tabular}

DE: disfunción eréctil.

estadísticamente significativo, pero el aspecto físico de la calidad vida sí mostró una correlación estadísticamente significativa $(p=0.004)$ (Tabla 3).

\section{Discusión}

La DE y la calidad de vida en los pacientes con HPB están bien descritas; sin embargo, en el presente trabajo se excluyeron los pacientes que presentaban alguna comorbilidad que afectara por sí misma la calidad de vida o favoreciera la DE, con el propósito de identificar la correlación directa entre ambas afecciones sin la interferencia de otras condiciones crónicas degenerativas. Por otra parte, el advenimiento de tratamientos nuevos, así como la evolución en el enfoque y la relevancia de la sexualidad, hacen necesaria la actualización de los reportes al respecto y la verificación en los pacientes.

Nuestros resultados coinciden con otros estudios al reportar que la DE afecta negativamente la percepción de la calidad de vida, sobre todo a expensas del ámbito físico $0^{1,2,5}$. Especialmente relevante es el 
Tabla 3. Correlaciones entre el resultado del cuestionario IIEF-5 y la calidad de vida en sus dos aparatados (salud física y salud mental)

\begin{tabular}{|c|c|c|c|c|c|}
\hline & & & $\begin{array}{c}\text { SF-12, } \\
\text { salud física }\end{array}$ & $\begin{array}{c}\text { SF-12, salud } \\
\text { mental }\end{array}$ & $\begin{array}{c}\text { Resultado } \\
\text { cuestionario IIEF-5 }\end{array}$ \\
\hline \multirow{9}{*}{$\begin{array}{l}\text { Rho de } \\
\text { Spearman }\end{array}$} & \multirow[t]{3}{*}{ SF-12, salud física } & Coeficiente de correlación & 1.000 & $-0.300^{*}$ & $0.285^{*}$ \\
\hline & & Significación (bilateral) & & 0.002 & 0.004 \\
\hline & & $\mathrm{n}$ & 101 & 101 & 101 \\
\hline & \multirow[t]{3}{*}{ SF-12, salud mental } & Coeficiente de correlación & $-0.300^{*}$ & 1.000 & 0.035 \\
\hline & & Significación (bilateral) & 0.002 & & 0.726 \\
\hline & & $\mathrm{n}$ & 101 & 101 & 101 \\
\hline & \multirow[t]{3}{*}{ Resultado cuestionario IIEF-5 } & Coeficiente de correlación & $0.285^{\star}$ & 0.035 & 1.000 \\
\hline & & Significación (bilateral) & 0.004 & 0.726 & \\
\hline & & $n$ & 101 & 101 & 101 \\
\hline
\end{tabular}

*La correlación es significativa con un valor de 0.01 .

Tabla 4. Salud mental y grado de disfunción eréctil

\begin{tabular}{|c|c|c|c|c|c|c|c|}
\hline & & \multicolumn{6}{|c|}{ Grado de disfunción eréctil } \\
\hline & & $\begin{array}{c}22-25 \\
\text { No hay DE }\end{array}$ & $\begin{array}{c}\text { 17-21 } \\
\text { DE leve }\end{array}$ & $\begin{array}{c}12-16 \mathrm{DE} \\
\text { leve- moderada }\end{array}$ & $\begin{array}{l}\text { 8-11 DE } \\
\text { moderada }\end{array}$ & $\begin{array}{c}5-7 \\
\text { DE grave }\end{array}$ & Total \\
\hline \multirow[t]{2}{*}{ Salud mental } & Por debajo de la media & 3 & 13 & 10 & 4 & 0 & 30 \\
\hline & Normal & 11 & 25 & 23 & 11 & 1 & 71 \\
\hline Total & & 14 & 38 & 33 & 15 & 1 & 101 \\
\hline
\end{tabular}

DE: disfunción eréctil

Tabla 5. Salud física y grado de disfunción eréctil

\begin{tabular}{llcccccc}
\hline & \multicolumn{3}{c}{ Grado de disfunción eréctil } & & \multicolumn{2}{c}{ Total } \\
\cline { 3 - 7 } & & $\begin{array}{c}\mathbf{2 2 - 2 5} \\
\text { No hay DE }\end{array}$ & $\begin{array}{c}\mathbf{1 7 - 2 1} \\
\text { DE leve }\end{array}$ & $\begin{array}{c}\mathbf{1 2 - 1 6} \\
\text { DE leve-moderada }\end{array}$ & $\begin{array}{c}\mathbf{8 - 1 1} \\
\text { DE moderada }\end{array}$ & $\begin{array}{c}\mathbf{5 - 7} \\
\text { DE grave }\end{array}$ \\
\hline \multirow{2}{*}{ Salud física } & Por debajo de la media & 6 & 22 & 24 & 9 & 1 & 62 \\
& Normal & 8 & 16 & 9 & 6 & 0 & 39 \\
\multirow{2}{*}{ Total } & 14 & 38 & 33 & 15 & 1 & 101 \\
\hline DE: disfunción eréctil. & & & &
\end{tabular}

estudio MALES, que divide la calidad de vida en sus aspectos mental y físico. Se encontró que, al calificar los niveles de satisfacción de calidad de vida, emergió un patrón: los hombres con DE describieron índices menores de satisfacción personal en todos los atributos evaluados de calidad de vida en comparación con los hombres sin $\mathrm{DE}$, en particular en vida sexual y salud en general ${ }^{14,15}$. Estos resultados son similares a los obtenidos en nuestro estudio, ya que los pacientes refirieron una disminución en la calidad de vida en el ámbito físico, pero no significativa en el ámbito mental (Tablas 4 y 5).

En España, en un estudio con 2476 pacientes a los que se aplicó el SF-36 para la estadificación de la calidad de vida, se encontró una disminución en el componente físico $(p=0.001)$, pero no en el componente mental ${ }^{16}$. El resultado anterior es similar al obtenido en el presente trabajo, en el cual la relación de 
la DE con el componente físico de calidad de vida mostró una $p=0.004$, pero sin ninguna relación entre la DE y el componente mental de calidad de vida, para el cual se obtuvo una $p=0.726$.

En cuanto al tratamiento médico y el promedio del puntaje IIEF-5 de DE encontramos similitud con trabajos previos, en los cuales se demuestra que el uso de terapia combinada con tamsulosina y finasterida obtiene promedios más bajos en el cuestionario IIEF-5 (mayor DE) que la tamsulosina sola, ${ }^{8,17,18}$. Por otro lado, en el estudio multinacional MALES, con un total de 27,839 pacientes, se halló que los grupos con DE con tratamiento y sin tratamiento no tuvieron diferencias significativas en los constructos de calidad de vida ${ }^{15}$.

La mayoría de los pacientes (38) de este estudio, según el cuestionario IIEF-5, se encontró con una DE leve. El promedio general de nuestra población obtenido por el cuestionario IIEF-5 fue de 16.30, clasificando a la población en $\mathrm{DE}$ leve, lo que debe diferenciarse de otros trabajos publicados en los que el promedio es más alto, debido quizá a que, como ya se comentó, en esta población se buscó que los pacientes no presentaran comorbilidad que pudiera favorecer la DE o mermar la percepción de la calidad de vida ${ }^{1,4,9,11,18-20}$.

\section{Conclusión}

Por lo anterior, se concluye que la HPB repercute en la DE y disminuye la calidad de vida en el aspecto físico, pero no en el aspecto mental.

\section{Conflicto de intereses}

Los autores declaran no tener ningún conflicto de intereses.

\section{Responsabilidades éticas}

Protección de personas y animales. Los autores declaran que los procedimientos seguidos se conformaron a las normas éticas del comité de experimentación humana responsable y de acuerdo con la Asociación Médica Mundial y la Declaración de Helsinki.

Confidencialidad de los datos. Los autores declaran que han seguido los protocolos de su centro de trabajo sobre la publicación de datos de pacientes.
Derecho a la privacidad y consentimiento informado. Los autores han obtenido el consentimiento informado de los pacientes y/o sujetos referidos en el artículo. Este documento obra en poder del autor de correspondencia.

\section{Bibliografía}

1. Kessler A, Sollie S, Challacombe B, Briggs K, Van Hemelrijck M. The global prevalence of erectile dysfunction: a review. BJU Int. 2019;124:587-99.

2. Alcaraz A, Carballido-Rodríguez J, Unda-Urzaiz M, Medina-López R Ruiz-Cerdá JL, Rodríguez-Rubio F, et al. Quality of life in patients with lower urinary tract symptoms associated with $\mathrm{BPH}$ : change over time in real-life practice according to treatment - the QUALIPROST study. Int Urol Nephrol. 2016;48:645-56.

3. Tong Y, Xie K, Li S. Self-care and quality of life in elderly Chinese patients with benign prostatic hyperplasia. Nurs Sci Q. 2020;33:79-84.

4. Skinder D, Zacharia I, Studin J, Covino J. Benign prostatic hyperplasia: a clinical review. J Am Acad Physician Assist. 2016;29:19-23.

5. De Nunzio C, Roehrborn CG, Andersson KE, McVary KT. Erectile dysfunction and lower urinary tract symptoms. Eur Urol Focus. 2017;3:352-63.

6. Mobley D, Feibus A, Baum N. Benign prostatic hyperplasia and urinary symptoms: evaluation and treatment. Postgrad Med. 2015;127:301-7.

7. Peyronnet B, Seisen T, Phé V, Misrai V, de la Taille A, Rouprêt M. Symptômes du bas appareil urinaire secondaire à une hyperplasie bénigne de prostate et dysfonction érectile: une revue systématique de la littérature. Press Medicale. 2017;46:145-53.

8. Calogero AE, Burgio G, Condorelli RA, Cannarella R, La Vignera S. Lower urinary tract symptoms/benign prostatic hyperplasia and erectile dysfunction: from physiology to clinical aspects. Aging Male. 2018;21:261-71.

9. Liu L, Zhao S, Li F, Li E, Kang R, Luo L, et al. Effect of $5 \alpha$-reductase inhibitors on sexual function: a meta-analysis and systematic review of randomized controlled trials. J Sex Med. 2016;13:1297-310.

10. Rezk MR, Tantawy MA, Wadie M, Weshahy SA. Smart spectrophotometric assessment of tamsulosin hydrochloride and tadalafil in their new pharmaceutical formulation for treatment of benign prostatic hyperplasia and erectile dysfunction. Spectrochim Acta - Part A Mol Biomol Spectrosc. 2020;227:117547.

11. Burnett AL, Nehra A, Breau RH, Culkin DJ, Faraday MM, Hakim LS, et al. Erectile dysfunction: AUA guideline. J Urol. 2018;200:633-41.

12. Hernández R, Thieme T. Adaptación y análisis psicométrico de la versión española del Î́ndice Internacional de Función Eréctil (IIEF) en población chilena. Terapia Psicológica. 2017;35:223-30.

13. Vilagut G, Ferrer M, Rajmil M, Rebollo P, Permanyer-Miralda G, Quintana JM, et al. El cuestionario de salud SF-36 español: una década de experiencia y nuevos desarrollos. Gac Sanit. 2005;19:135-50.

14. Diagnóstico y tratamiento de la hiperplasia prostática benigna. México: Secretaría de Salud; 2009. (Consultado el 26 de mayo de 2020.) Disponible en: http://www.cenetec.salud.gob.mx/descargas/gpc/CatalogoMaestro/176_GPC_HIPERPLASIA_PROSTATICA/Gpc_hipertrofia_prostatica.pdf.

15. Sand MS, Fisher W, Rosen R, Heiman J, Eardley I. Erectile dysfunction and constructs of masculinity and quality of life in the multinational Men's Attitudes to Life Events and Sexuality (MALES) study. J Sex Med. 2008;5:583-94.

16. Sánchez-Cruz JJ, Cabrera-León A, Martín-Morales A, Fernández A, Burgos R, Rejas J. Male erectile dysfunction and health-related quality of life. Eur Urol. 2003;44:245-53.

17. Favilla V, Russo GI, Privitera S, Castelli T, Giardina R, Calogero AE, et al. Impact of combination therapy 5-alpha reductase inhibitors (5-ARI) plus alpha-blockers $(A B)$ on erectile dysfunction and decrease of libido in patients with LUTS/BPH: a systematic review with meta-analysis. Aging Male. 2016;19:175-81.

18. Calogero AE, Burgio $G$, Condorelli RA, Cannarella R, La Vignera S. Treatment of lower urinary tract symptoms/benign prostatic hyperplasia and erectile dysfunction. Aging Male. 2018;21:272-80.

19. Zhao H, Kim HH. The complex relationship between lower urinary tract symptoms and sexual health. Curr Urol Rep. 2019;20:58.

20. Li Z, Chen P, Wang J, Mao Q, Xiang H, Wang X, et al. The impact of surgical treatments for lower urinary tract symptoms/benign prostatic hyperplasia on male erectile function. A systematic review and network meta-analysis. Medicine (Balt). 2016;95:e3862. 